\title{
Analysis and Discussion on QoS Mechanism in IP Broadband Networks
}

\author{
Yu Jianhua \\ Chongqing Technology and Business Institute, Chongqing 401520, China
}

Keywords: QoS, mechanism, IP broadband networks

\begin{abstract}
With the increase of video, image, music and other multimedia services in the network, telecom operators need to improve network performance and experience quality of telecom services, so as to enhance their competitiveness. It is particularly important to rationally deploy network QoS (Quality of Service). This paper introduces what is QoS and the current situation of QoS in broadband access network, and some effective mechanisms for how to guarantee QoS is proposed in this paper, and finally from the perspective of development, discussed in the broadband access network implementation of the main ideas and related technologies of QoS.
\end{abstract}

\section{Introduction}

With the advancement of science and technology and the continuous improvement of people's demand, the traditional communication services are expanding to the fields of information entertainment and digital life. IPTV, VoIP and P2P applications are becoming more and more abundant. As a result, the services carried by broadband networks will gradually develop from traditional Internet services to multi-services. This requires the implementation of broadband network transformation to adapt to the changes in business needs and business development requirements, whether the successful implementation of network transformation involves a number of factors, such as: the physical structure of the network, the type of network services, QoS capabilities, operational support systems. Among them, network business provision and QoS capability are the most basic factors.

QoS is a control mechanism, which provides different priorities for different users or different data streams, or according to the requirements of the application, to ensure that the performance of the data stream to a certain level. Normally, if the network is only used for a specific, time-free application, QoS is not required, such as Web applications, or E-mail settings. But it is necessary for key applications and multimedia applications. When the network is overloaded or congested, QoS can ensure that important traffic is not delayed or discarded, while ensuring the efficient operation of the network. QoS guarantees are very important for networks with limited capacity, especially for streaming multimedia applications, such as VoIP and IPTV, which often require a fixed transmission rate and are sensitive to delay.

\section{QoS mechanism of IP broadband network}

The current broadband access network mainly carries data services, but also a small number of video streaming services, all services can not be guaranteed QoS, the network for all services to provide only a best-effort forwarding service. For the private line business, it is generally provided by ATM, SDH, MSTP and DDN network. Early metropolitan area network/access network devices have uneven capabilities in QoS guarantee mechanisms such as DiffServ/802.1p priority. However, they lack operational telecommunication-level QoS schemes and are unable to achieve end-to-end QoS guarantee, resulting in intermittent video, good and bad, VoIP services often appear packet loss, and users can not enjoy multimedia to the full. IP business experience.

In order to implement QoS on the network, the prerequisite is to have accurate prediction, that is, before the user's packet flow enters the network, a network resource determination mechanism is adopted to determine whether the network can provide sufficient resources, which is equivalent to the user and the network provider to develop a contract to achieve QoS. When the contract is 
reached, the network provider must set the QoS parameters of each node. At the same time, the network node must provide a monitoring and scheduling mechanism. The monitoring program will monitor the flow of packets sent to the network node by the user. If the packets exceed the contract content, they will be discarded or the QoS level will be degraded. Scheduling is to give different priorities to competitive packets depending on the QoS level. To meet the different needs of QoS, there are the following QoS protocols and algorithms.

\subsection{RSVP - Resource Reservation Protocol}

RSVP is a signaling protocol that provides resource reservation for establishing connections, controls integrated services, and often provides simulation circuits in IP networks. RSVP is the most complex of all QoS technologies, it is most different from the best-effort IP service standard. It provides the highest QoS level, guarantees services, quantifies resource allocation, and feeds back subtle changes in quality of service to QoS-enabled applications and users.

\subsection{MPLS - Multi-Protocol label switching}

MPLS refers to the technology of label switching, which is similar to ATM switching technology, to transmit IP packets to destination. When an IP packet enters the MPLS domain, the LER (Label Edge Router) first checks the IP packet header that enters the MPLS network, then finds out the corresponding service level marker according to the IP packet header, then pastes the IP packet with this marker and sends it into the MPLS network area; the LSR (Label Switch Router) located in the MPLS network receives it. When a labeled IP packet is attached, the LSR can transfer the packet to its destination by hardware according to the labeled value of the packet.

\subsection{Differentiated services - DiffServ priority ranking}

Differential services provide a simple and crude way to categorize services. At present, two most representative service levels (business categories) have been stipulated:

Fast forwarding (EF): there is a separate code point (DiffServ value). EF can minimize delay and jitter, thus providing the highest level of service quality. Any business beyond the scope of service is deleted.

Guaranteed forwarding (AF): there are four levels, each level has three descending processes (total of 12 code points). Traffic beyond the AF range will not be transmitted as high as possible in the "business scope" of the business.

\section{How to implement QoS solution in broadband access network}

\section{1 change the network structure}

The typical broadband network can be divided into three layers: access layer, convergence layer and core layer. Access layer provides XDSL and many other access modes, such as Internet interconnection, voice, video and other services. The service flow of convergence layer devices to access layer through FE / GE, ATM155 and other interfaces converges to the core layer of MAN through GE / POS, and finally converges from the core devices of MAN to the backbone network. In the traditional tree topology broadband access network, the upstream traffic of different services converges in the upstream direction, and the quality of service of the upstream traffic of various services needs to be guaranteed. For the downlink direction, BAS, as the service control point, also has the convergence phenomenon of downlink traffic for different services, especially when different traffic comes from different backbone networks. In addition, broadband access networks may use data links at different rates, resulting in rate mismatches and short-term congestion. Therefore, downlink traffic also needs to ensure quality of service. Therefore, the upstream and downstream directions of broadband access network need to be implemented with QoS solution.

\subsection{The overall planning principles of QOS}

To implement broadband access to QoS, we must first carry out the master plan of QoS, and its planning principles can be as follows: 
(1) According to the characteristics of different services in the access network for the implementation of business types of service shunting, the types of services can be considered at present can be divided into VoIP voice, video, dedicated line interconnection and Internet access. In addition, it must be considered that there are management and control signaling in the broadband network itself, which has the same QoS guarantee requirements as the VoIP data stream.

(2) Implement the service VLAN strategy in the convergence layer of the access network, and make reasonable bandwidth planning for the service VLAN according to the service and traffic characteristics.

(3) Different customers implement different QoS: For important enterprise VPN lines and interconnected customers in the same city, at least in the access network convergence layer, the implementation of exclusive bandwidth and independent logical channel (VLAN) two-tier QoS strategy; for individual and household users, according to the user's business type, provide different QoS strategy.

(4) Different types of services implement different QoS: VoIP, video and Internet access are the main services for individual and household users, and the priority of services is from high to low. The absolute priority scheduling strategy is implemented between these three types of services; and Internet access services can be further divided into two parts according to the content downstream. Class priority: Internet services with certain QoS guarantees and ordinary Internet services without QoS guarantees, which implement weighted average scheduling strategy.

\section{3 reasonable deployment of QoS}

The deployment of QoS technology mainly includes resource control, resource isolation and resource scheduling. Resource control restricts the use of network resources, mainly through traffic licensing control to achieve, such as restrictions on different types of traffic, traffic priority control and so on, resource constraints make the different types of traffic in the network have a reasonable proportion and distribution. Resource isolation mainly guarantees that different traffic does not interfere with each other. This isolation can be achieved by setting traffic priority. Priority mainly ensures that low-priority traffic does not interfere with high-priority traffic. Resource scheduling assigns and schedules resources.

The network model shown in Figure 1 is a basic DiffServ architecture model.

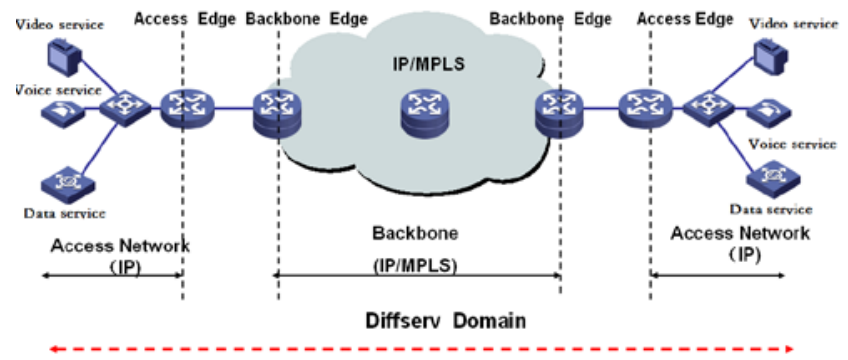

Fig. 1 DiffServ architecture model

Diffserv architecture is generally divided into two levels, access layer, backbone layer, backbone area for IP or MPLS forwarding. According to the end-to-end deployment principle, QoS policies are deployed in the following management areas: access network, access network edge, backbone network edge, backbone network.

The implementation of IP QOS for the above network models generally involves the following 6 steps:

(1) identify key applications in business and categorize key applications. Before classifying applications, it is necessary to clarify the delay, packet loss rate, jitter requirements, application properties or performance requirements, and transport layer protocol stack used by these key applications.

(2) markup, setting DSCP/IP Precedence priority markup for different business data streams.

(3) define the QOS regulatory strategy for each type of business or application. Network bandwidth (including minimum bandwidth, maximum bandwidth, burst rate, etc.) is allocated for 
different traffic data streams according to the classification and labeling information of traffic data streams.

(4) Queuing: queuing the output traffic data stream to achieve priority transmission of key traffic;

(5) congestion avoidance (WRED): dispose of network congestion by dropping low priority packets.

(6) Monitor the delay, jitter and packet loss rate of key applications, and adjust the QOS strategy to ensure the application performance according to the traffic in the network.

\subsection{Optimize the existing QoS mode}

In view of the deployment requirements of broadband access QoS, the following five aspects should be taken into consideration to implement the technical transformation of broadband access network in order to solve the QoS technical problems faced by the access network adapting to multi-service bearers:

(1) optimize the broadband access network architecture. The BRAS is moved down, the level of convergence network is reduced, the large-scale two-tier convergence network is changed into several smaller two-tier networks which are isolated from each other, the cascade series of DSLAM is reduced as far as possible, and the bandwidth capability of the upper interface of DSLAM devices is expanded.

(2) DSLAMIP transformation. To support video services, existing ATM uplink DSLAM devices must be transformed into IP upstream, or IP-DSLAM nodes must be built next to the original ATM-DSLAM nodes to cut the multi-service users directly to the new IP-DSLAM nodes.

(3) multi service VLAN isolation. In the aggregation network, different VLAN strategies are implemented for different services to diversify services and ensure the isolation between VLANs.

(4) the unification and upgrading of QoS functions of network devices. The QoS function of network equipment in access network is planned in a unified way, especially the three key components of BRAS, access terminal and access platform, and the corresponding technical upgrade or transformation is carried out to ensure the support of network equipment to end-to-end QoS function in access network.

(5) intelligent multi service port access terminal. Intelligent multi-service port access terminal is used to replace the existing single-port terminal, and multi-PVC uplink, implementation of business and port, port and PVC binding strategy.

\section{Conclusion}

With the rapid development of network technology, the traditional "Best effort " data forwarding mechanism can not meet the requirements of users. How to provide QoS support for broadband network has become the focus of attention of telecom operators. This paper expounds the architecture of QoS in IP network, analyzes several commonly used QoS mechanisms, and puts forward some optimization suggestions for the deployment of QoS mechanism in broadband network.

\section{References}

[1] LIN Chuang, WANG Yuan-Zhuo, REN Feng-Yuan. Research on QoS in Next Generation Network[J]. Chinese Journal of Computers, 2008, 31(9):1525-1535.

[2] NIU Ying-xia, HE Xin. Development strategy of home broadband IPTV service[J.] Telecommunications Science, 2017, 33(4):163-169.

[3] FENG Ping, YUAN Liang. Precise Recognition of MPLS L2VPN QoS[J]. Communications Technology, 2015, 48(3):342-345.

[4] JIN Wei-chao. Implementation of specific service quality of QoS based on DiffServ system[J]. 2018, 4:132-135. 